\title{
Determinación de factores trombofílicos en pacientes con trombosis venosas retinianas
}

\author{
Leonidas Traipe $\mathbf{C}^{\mathbf{1}}$, Guillermo Conte $\mathbf{L}^{2}$, \\ Francisco J Conte $S^{3 a}$, Santiago Ibáñez $L^{3}$, \\ Patricio Meza $\mathbf{R}^{\mathbf{1}}$, Basilio Rojas $\mathbf{U}^{\mathbf{3}}$, \\ Marianela Cuneo $\mathrm{V}^{2, b}$, Juan Verdaguer $\mathrm{T}^{3}$. \\ Markers of thrombophilia in patients \\ with retinal vein thrombosis
}

Background: Thrombophilia is an alteration of hemostasis that increases the risk to venous or arterial thrombosis. This condition may be the underlying cause of retinal vein thrombosis. Aim: To study the presence of thrombophilia in patients with retinal vein thrombosis. Patients and methods: Prospective study of 55 patients aged 22 to 86 years, with retinal vein thrombosis (central or branch). Antithrombin III, coagulant protein C, functional protein S, resistance to activated C protein, homocysteine, prothrombin G20210A gene, lupus anticoagulant and anticardiolipin antibodies were measured in all. Results: Seventeen patients had thrombophilic markers (antiphospholipid syndrome in seven, hyperhomocysteinemia in six and resistance to protein $\mathrm{C}$ in three). Of these 17 patients, 53\% had high blood pressure, 35\% an abnormal serum lipid profile and $23 \%$ a personal history of thrombosis. The thrombosis was central in 12 (ischemic in four) and of a branch in five (ischemic in two). Conclusions: Thrombophilic markers must be assessed in patients with retinal vein thrombosis (Rev Méd Chile 2005; 133: 167-74).

(Key Words: Activated protein C resistance; Antiphospholipid syndrome; Retinal vein occlusion; Thrombophilia)

\footnotetext{
Recibido el 1 de marzo, 2004. Aceptado en versión corregida el 16 de diciembre, 2004.

${ }^{1}$ Servicio de Oftalmología y de ${ }^{2}$ Hematología, Hospital Clínico de la Universidad de Chile. ${ }^{3}$ Fundación Oftalmológica Los Andes, Santiago.

aMédico Becario en Oftalmología, Universidad de Los Andes.

${ }^{\mathrm{b}}$ Tecnólogo Médico.
}

L as oclusiones venosas de retina constituyen una de las vasculopatías retinianas más frecuentes. Se observan, mayoritariamente, en personas de edad, y minoritariamente (10-15\%) se presentan en pacientes jóvenes ${ }^{1}$. Los factores predisponentes pueden ser sistémicos u oculares.

Dentro de los sistémicos están: edad avanzada, hipertensión arterial (HTA), diabetes mellitus (DM) y síndrome de hiperviscosidad, que en forma aislada puede manifestarse como una oclusión venosa retiniana ${ }^{2-5}$. Es importante destacar

Correspondencia a: Dr. Leonidas Traipe C. Fundación Oftalmológica Los Andes. Las Hualtatas 5951. Vitacura. Santiago de Chile. E-mail: ltraipe@entelchile.net que dichos factores de riesgo sistémicos son inespecíficos, porque son similares a los factores de riesgo de trombosis arterial.

Por su parte, los factores predisponentes oculares o locales son: presencia de presión intraocular elevada, hipermetropía, anomalías vasculares oculares congénitas y periflebitis retinal. Ninguna de ellas es determinante de trombosis ${ }^{6,7}$.

Existen dos tipos principales de oclusiones vasculares venosas retinales (OCVR): la de vena central y la de rama venosa.

La primera, según las manifestaciones clínicas y el pronóstico, se divide en OCVR isquémica y no isquémica y ambas provocan una muy mala 
agudeza visual (AV). No obstante, la isquémica se diferencia porque puede presentar una lesión de la aferencia de la vía óptica, que se reconoce por la presencia de una alteración en los reflejos pupilares (defecto pupilar aferente relativo), una mayor cantidad de hemorragias, manchas algodonosas, edema del disco óptico y gran tortuosidad venosa al fondo de $0 j 0^{8}$. En el examen conocido como angiografía con fluoresceína, que ayuda a evaluar la circulación retinal, la OCVR isquémica muestra extensas zonas de no perfusión capilar; y en el electrorretinograma (ERG) puede aparecer una disminución de la onda $\mathrm{b}$. Todo esto determina un peor pronóstico visual y la posibilidad de desarrollar complicaciones como hemorragia vítrea o glaucoma neovascular.

Por otro lado, desde hace 30 años se han descrito alteraciones retinianas en el Lupus Eritematoso Sistémico (LES), de las que la más importante es la presencia de trombosis de la arteria central de la retina (ACR) y la vena central de la retina $(\mathrm{VCR})^{9}$. Ambos casos se presentan con mayor frecuencia cuando se asocian al síndrome antifosfolípido (SAF), especialmente cuando en éste hay presencia de anticuerpos anticardiolipinas y, en menor proporción, cuando hay anticoagulante lúpico ${ }^{10-12}$. El SAF es parte de una condición clínica llamada trombofilia, que puede definirse, en general, como una predisposición, hereditaria o adquirida, para la aparición de trombosis venosa recurrente que, en ocasiones, se presenta en sitios inusuales del organismo ${ }^{13-16}$.

Una primera causa de trombofilia es el déficit de anticoagulantes o inhibidores naturales de la coagulación, como la Antitrombina (AT), Proteína C (PC) y Proteína S (PS) ${ }^{17,18}$.

Una segunda es la resistencia de la proteína $C$ activada (RPCa), provocada por una mutación de un aminoácido en el Factor V, lo que determina una proteólisis lenta de éste por acción del complejo PC-PS ${ }^{19-21}$. Cuando el aminoácido mutado corresponde a la posición 506 Arg-Glutámico se conoce como Factor $\mathrm{V}$ Leiden y se puede determinar por medio de $\mathrm{PCR}^{22}$.

Una tercera causa de trombofilia es la aparición de un mayor efecto procoagulante de factores de la coagulación, como la mutación del gen de la protrombina $\mathrm{G} 20210 \mathrm{~A}^{23,24}$.

Una cuarta, de menor frecuencia, se debe a un aumento de los niveles o función de los factores de la coagulación VIII, IX, XI; o de la lipoproteína A; o a una disfibrinogenemia; o a una deficiencia de plasminógeno, o a un aumento de los niveles del inhibidor de la fibrinolisis activado por trombina, conocido como TAFl ${ }^{25-27}$.

Finalmente, una quinta causa de esta predisposición a trombosis se ha asociado al aumento de la homocisteína plasmática que, en un tercio de los casos, está determinada por una mutación de la metiltetrahidrofolatorreductasa (MTHF) C677T que, con los anticuerpos asociados al SAF, son los únicos factores causantes de trombosis venosa y también arterial ${ }^{24,28-31}$.

Existen publicaciones en que algunos casos de trombofilia se asociaron a aumento de PAI-1 (inhibidor del activador del plasminógeno) pero, en la actualidad, se sabe que forma parte del síndrome $\mathrm{X}$ (o síndrome de resistencia a la insulina), que se asocia a la hipertensión arterial, a la dislipidemia, y a la aparición de diabetes mellitus, todos factores sistémicos frecuentes en OCVR 32,33 .

La aparición de trombofilia como etiología de trombosis retiniana venosa y arterial, hace necesario, por tanto, considerar su estudio, ya que puede dar lugar a diferentes acciones terapéuticas.

\section{MATERIAL Y MÉTODOS}

En un estudio prospectivo observacional en que participaron los servicios de oftalmología del Hospital Clínico de la Universidad de Chile, la Fundación Oftalmológica Los Andes, el Instituto Oftalmológico Profesor Arentsen (IOPA) y el Laboratorio de Hematología del Hospital Clínico de la Universidad de Chile, se estudiaron pacientes derivados por diagnóstico de trombosis venosa central o de rama, sin etiología precisa, en el período marzo 2001-noviembre 2003.

En todos los pacientes se investigó la presencia de antecedentes personales o familiares de trombosis, hipertensión arterial, diabetes mellitus, dislipidemia, enfermedades sistémicas como cáncer, 0 uso de anticonceptivos orales.

El estudio de laboratorio incluyó medición de antitrombina III (AT III), por método cromogénico (Dade Behring) en un espectrofotómetro PerkinElmer; proteína C coagulante (PCc) y proteína S funcional (PSf), por método coagulométrico (Dade Behring); resistencia a la proteína $\mathrm{C}$ activa- 
da (RPCa) por método coagulométrico (Grifols), en un equipo Behring Fibrintimer A, y Factor V Leiden por PCR; homocisteína plasmática, por inmunoanálisis de polarización de la fluoresceína (Abbott), en un analizador IMx; gen de la protrombina G20210A, por PCR; anticoagulante lúpico (Grifols) en un equipo Behring Fibrintimer A; y anticuerpos anticardiolipina IgM-IgG, por método Elisa (Grifols). La determinación de la mutación en la enzima metiltetrahidrofolato reductasa (MTHFR), solicitada en casos de hiperhomocisteinemia, se realizó mediante PCR en el laboratorio de la Universidad Católica de Chile.

Desde el punto de vista ocular, se efectuaron como mínimo cuatro evaluaciones clínicas, una al iniciar el estudio y, otras tres, cada 30 días. En ellas, se incluyeron los siguientes elementos: anamnesis próxima y remota (al ingreso), toma de agudeza visual en cartilla Snellen, tonometría aplanática, biomicroscopia, gonioscopia con lente de tres espejos (Goldmann) y fondo de ojo bilateral en midriasis farmacológica con Tropica- mida al 1\%. A cada paciente se le solicitó además una angiografía retinal con fluoresceína.

\section{RESULTADOS}

Se estudiaron 55 pacientes con trombosis retiniana sin evidencia precisa de etiología. De ellos, se encontró asociada etiología trombofílica sólo en 17 casos, 11 mujeres y 6 hombres, todos con un promedio de edad de 60 años (30-85). De las mujeres, $81 \%$ tenía más de 50 años; $66,6 \%$ de los hombres eran menores de 50.

El grupo sin etiología trombofílica asociada al cuadro trombótico (esto es, los 38 pacientes restantes), tenía un promedio de edad de 62 años (22-86) y $76,5 \%$ presentaba una oclusión de vena central. De los factores de riesgo sistémico de trombosis venosa retinal, $58,8 \%$ tenía hipertensión arterial; 29,4\% dislipidemia; y 5,8\% diabetes mellitus.

El estudio de factores sistémicos asociados a la etiología trombofílica se muestra en la Tabla

Tabla 1. Factores asociados a las trombofilias

\begin{tabular}{|c|c|c|c|c|c|c|c|}
\hline Caso & Factor asociado & $\mathrm{HH}$ & $\mathrm{SAF}$ & FV-Leiden & G20210a & Def PS & Def PC \\
\hline 1 & No & & & & & + & \\
\hline 2 & Dislipidemia & & + & & & + & \\
\hline 3 & Ateromatosis carotidea bilateral & & + & & & & \\
\hline 4 & $\begin{array}{l}\text { Hx Fliar trombosis, dislipidemia, } \\
\text { C. coronaria }\end{array}$ & & + & & & & \\
\hline 5 & Hx personal trombosis, HTA + & + & + & & & & \\
\hline 6 & Hx personal trombosis, HTA + & & & + & & & \\
\hline 7 & Hx personal trombosis, dislipidemia & & & + & & & \\
\hline 8 & $\mathrm{HTA}+, \mathrm{ACO}$ & & & & & & + \\
\hline 9 & Dislipidemia, ACO & & & & & + & \\
\hline 10 & Hx personal trombosis, HTA + & & & & + & + & \\
\hline 11 & $\mathrm{HTA}+, \mathrm{DM}+$, cáncer & + & & & & & \\
\hline 12 & $\mathrm{HTA}+, \mathrm{DM}+$, C. coronaria & & & + & & & \\
\hline 13 & $\mathrm{HTA}+, \mathrm{DM}+$ & + & & & & & \\
\hline 14 & HTA +, dislipidemia & + & & & & & \\
\hline 15 & No & & + & & & & \\
\hline 16 & No & + & + & & & & \\
\hline 17 & HTA +, dislipidemia, glaucoma & + & + & & + & & \\
\hline
\end{tabular}

HH: hiperhomocisteinemia; SAF: síndrome antifosfolípido; G20210A: mutación del gen de la protrombina G20210A; Def PS: deficiencia proteína S; DEF PC: deficiencia proteína C; Hx Fliar: historia familiar; C. coronaria: cardiopatía coronaria; HTA: hipertensión arterial; DM: diabetes mellitus. 


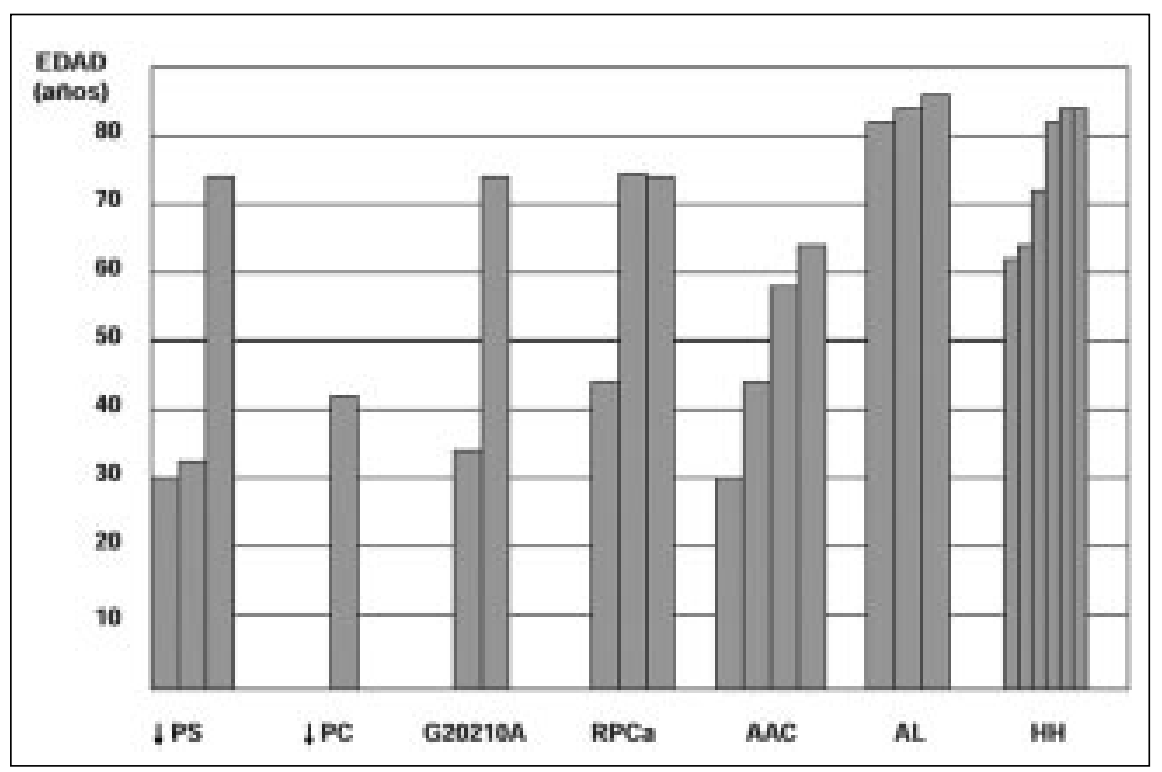

FiguRA 1. Distribución de trombofilias por edad. PS: Proteína S; PC: Proteína C; G20210A: Mutación del gen de la protrombina G20210A; RPCa: Resistencia a la proteína $C$ activada; AAC: Anticuerpos anticardiolipinas; $\mathrm{AL}$ : Anticoagulante lúpico; $\mathrm{HH}$ : Hiperhomocisteinemia.

1. De ellos, la mayoría se asocian a factores de riesgo de trombosis arterial como hipertensión arterial (53\%), dislipidemia (35\%), diabetes mellitus $(17,6 \%)$, y, en menor proporción, a antecedentes personales de trombosis o cáncer, propios de las trombosis venosas. Excepcionalmente, en un caso se demostró un factor local: glaucoma.

Las trombofilias detectadas se describen en la Tabla 1. De ellas, las más frecuentes fueron síndrome antifosfolípido, en 7 casos (4 con anticuerpos anticardiolipina y 3 con anticoagulante lúpico), hiperhomocisteinemia en 6 (uno con mutación MTHFR +) y RPCa-Factor V Leiden en 3. De los 17 pacientes con trombofilia, 5 de ellos presentaban trombofilia combinada (Tabla 1).

Al estudiar las trombofilias por edad, las de tipo únicamente hereditario, como la deficiencia de PC o PS, mostraron una tendencia (no estadísticamente significativa) a presentarse en grupos etarios menores, mientras que las adquiridas, como SAF o hiperhomocisteinemia, en grupos etarios mayores (Figura 1).

Desde el punto de vista oftalmológico, se presentaron 12 casos de trombosis de vena central de retina y 5 de rama, con 1/3 de casos isquémi-

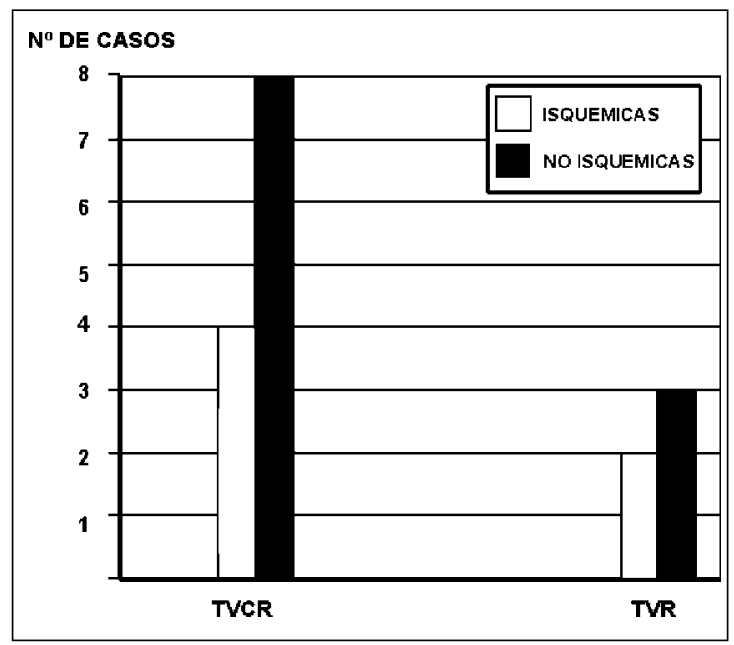

FIGURA 2. Tipos de trombosis asociadas a trombofilias. TVCR: Trombosis de vena central de retina; TVR: Trombosis de rama venosa retinal.

cos en las de vena central (Figura 2). De las trombosis isquémicas su distribución por sexo y etiología se aprecia en la Tabla 2.

Las características visuales de los casos más graves con trombosis, se resumen en la Tabla 3. 
DisCUSIÓN

El concepto clásico de trombofilia ha variado con los últimos descubrimientos en esta área. Así puede definirse como una condición clínica en que existe una mayor propensión, hereditaria 0 adquirida, a la aparición de trombosis venosa o arterial debido a una anormalidad en la hemostasis. En general, en la literatura, dicha patología se expresa antes de los 50 años, pero en casos de trombosis venosa retiniana (central o de rama), puede presentarse a cualquier edad $^{34}$. Esto último, se ajusta a procesos adqui-

Tabla 2. D istribución de trombosis retiniana por sexo y etiologías

\begin{tabular}{|lcc|}
\hline $\begin{array}{l}\text { Trombosis } \\
\text { isquémicas }\end{array}$ & TVCR & TVR \\
\hline Hombres & 2 & 1 \\
Mujeres & 2 & 1 \\
Etiologías & $\begin{array}{c}\text { Hiperhomocisteina (3)* } \\
\text { A lúpico } \\
\end{array}$ & $\begin{array}{c}\text { RPCa } \\
\text { Autación G20210A }\end{array}$ \\
\hline
\end{tabular}

TVCR: Trombosis de vena central de retina; TVR: Trombosis venosa de rama; RPCa: Resistencia a la proteína C; Mutación G20210A: mutación gen de la protrombina G20210A. ridos como el síndrome antifosfolípido o la hiperhomocisteinemia.

Esta serie clínica de 55 pacientes no tiene por objeto determinar la prevalencia de trombofilia en pacientes que padecen trombosis venosa retiniana. El alto número de casos del universo estudiado puede estar determinado por los antecedentes de trombosis familiar o personal que claramente orientan a trombofilia.

Las principales causas de trombosis venosa retiniana fueron el síndrome antifosfolípido, la hiperhomocisteinemia, la RPCa o Factor V Leiden (Tabla 2). Estos resultados coinciden con la literatura, la cual, en su mayoría se refiere a casos de trombosis venosa retiniana asociada a Factor $\mathrm{V}$ Leiden o Síndrome Antifosfolípido 10,22,35,36, lo que contrasta con etiologías más infrecuentes de carácter hereditario como la deficiencia de Proteína $\mathrm{C}$ o $\mathrm{S}$.

Existen trabajos recientes que describen la presencia de uno o más factores asociados sistémicos o locales en trombosis venosas retinianas ${ }^{2}$. En éste, los más relevantes fueron: la hipertensión arterial con 9 de los 17 casos, dislipidemia en 6 y diabetes mellitus en 3. Es de particular importancia investigar los antecedentes de trombosis venosas previas (4 casos) o de factores desencadenantes como: uso de anticonceptivos orales ( 2 casos de las 10 mujeres), el reposo prolongado, una cirugía reciente y trauma o embarazo, porque aumentan el riesgo de trombosis en pacientes con trombofilia (Tabla 1).

Tabla 3. C aracterísticas oftalmológicas de los pacientes con trombosis venosas retinianas de tipo isquémico

\begin{tabular}{|rcccl|}
\hline $\begin{array}{c}\text { Caso } \\
\mathrm{N}^{\circ}\end{array}$ & $\begin{array}{c}\text { Tipo } \\
\text { trombosis }\end{array}$ & $\begin{array}{c}\text { AV } \\
\text { inicial }\end{array}$ & $\begin{array}{c}\text { AV } \\
\text { final }\end{array}$ & Complicación \\
\hline 1 & TVCR & $\begin{array}{c}20 / 400 \\
\text { a } 1 \mathrm{mt}\end{array}$ & $20 / 200$ & GNV \\
5 & TVCR & $\operatorname{lmp}$ & $\operatorname{lmp}$ & - \\
11 & TVCR & cds & $\operatorname{lbp}$ & Hemorragia vítrea \\
12 & TVR & $20 / 40$ & $\operatorname{lbp}$ & Hemorragia vítrea \\
13 & TVCR & $20 / 400$ & $20 / 400$ & - \\
16 & TVR & $20 / 400$ & $20 / 400$ & Edema macular \\
\hline
\end{tabular}

TVCR: Trombosis de vena central de retina; TVR: Trombosis venosa de rama; lmp: luz mala proyección; cds: cuenta dedos; GNV: Glaucoma neovascular. 
La etiología trombofílica en trombosis venosa de retina debe considerarse en todo paciente por las siguientes razones:

1. Existencia de base de un estado de hipercoagulabilidad que hace más probable la aparición de trombosis especialmente cuando existe un factor de trombofilia. De hecho, en pacientes con OCVR se ha demostrado elevación de fragmento $1+2$ de la protrombina, factor VII activado y Dímero $\mathrm{D}^{37}$. 2. La presencia de factores sistémicos puede asociarse a trombofilias, que se han descubierto recientemente, y se ignora con precisión su frecuencia real. Sin embargo, es importante investigarlas cuando existen factores desencadenantes de trombosis venosa como: reposo prolongado, cirugía, embarazo y uso de anticonceptivos. 3. Algunas trombofilias son susceptibles de tratamiento. Así, los casos de hiperhomocisteinemia y mutación de MTHFR pueden mejorar con la administración de complejo B (B6 y B12) y ácido fólico. En otras situaciones, como el síndrome antifosfolípido, se ha utilizado ticlopidina, heparina de bajo peso molecular o ambos. No existen publicaciones que demuestren la necesidad de

\section{REFERENCIAS}

1. Prisco D, Marcuca R. Retinal vein trombosis: risks factors, pathogenesis and therapeutic approach. Pathophysiol Haemost Thromb 2002; 32: 308-11.

2. Hayreh SS, Zimmerman B, Mc Carthy MJ, Podhajsky P. Systemic diseases associated with various types of retinal vein occlusion. Am J Ophthalmol 2001; 131: $61-77$

3. Fong A, Schatz H. Central retinal vein occlusion in young adults. Surv Ophthalmol 1993; 37: 393-417.

4. Arend O, Remky A, Jung F, Kiesewetter H, Reim M, Wolf S. Role of rheologic factors in patients with acute central retinal vein occlusion. Ophthalmology 1996; 103: 80-6.

5. Bernard AG, Chanabal A, Le Long F. Elevated erythrocyte aggregation in patients with central retinal vein occlusion and without conventional risk factors. Ophthalmology 1994; 101: 1483-7.

6. The Eye Disease Case-Control Study Group. Risks factors for central retinal vein occlusion. Arch Ophthalmol 1996; 114: 545-54. usar tratamientos antitrombóticos o anticoagulantes en todos los pacientes.

Es importante resaltar que en este trabajo se estudiaron simultáneamente las trombofilias más importantes, porque ha sido una limitante de varias publicaciones que sólo incluyen causas aisladas.

Ante la presencia de cualquier factor de trombofilia existe la ineludible necesidad de efectuar un estudio de laboratorio completo. Ello, porque en $25 \%$ de los casos los factores, hereditarios o adquiridos, son múltiples ${ }^{38}$ y con una supraadición que eleva el riesgo de trombosis. En nuestro trabajo, cerca de $30 \%$ de los casos presentaban una trombofilia combinada, lo que concuerda con lo descrito en la literatura.

Por todo lo anterior, el estudio de trombofilias debe iniciarse con las causas más frecuentes: como síndrome antifosfolípido, hiperhomocisteinemia y resistencia a la proteína $\mathrm{C}$ activada. Además tienen que descartarse factores sistémicos como: hipertensión arterial y antecedentes de trombosis previas para poder aplicar un tratamiento racional ante la presencia de todos estos factores etiológicos.

7. Rath E, Franck R, Shin D, Kim CH. Risks factors for retinal vein occlusions: a case control study. Ophthalmology 1992; 99: 509-14.

8. The Central Vein Occlusion Study Group. Natural history and clinical management of central retinal vein occlusion. Arch Ophthalmol 1997; 115: 48691.

9. Kieiner RC, Najarian LV, Schatten S, Jabs DA, Patz A, KaPLAN HJ. Vaso-occlusive retinopathy associated with anti-phospholipid antibodies (lupus anticoagulant retinopathy). Ophthalmology 1989; 896-904.

10. Cobo-Soriano R, Sánchez-Ramón S, Aparicio MJ, Tejeiro MA, Vidal P, Sú́rez-Leoz M et al. Antiphospholipid antibodies and retinal thrombosis in patients without risk factors: a prospective case-control study. Am J Ophthamol 1999; 128: 725-32.

11. Wiehens B, Schroder J, Potzsch B, Rochels R. Primary antiphospholipid antibody syndrome and retinal occlusive vasculopathy. Am J Ophthalmol 1997; 123: 848-50. 
12. Glacet-Bernard A, Bayani N, Chretien P, Cochard C, LELONG F, Coscas G. Antiphospholipid antibodies in retinal vascular occlusions. Arch Opthalmol 1994; 112: 790-5.

13. Crowther MA, Kelton JG. Congenital thrombophilic states associated with venous thrombosis: a qualitative overview and proposed classification. Ann Intern Med 2002; 138: 128-34.

14. BAUER KA. The trombophilias: well defined risks factors with uncertain therapeutics implications. Ann Intern Med 2001; 135: 367-73.

15. Chak M, Walace GR, Graham EM, Stanford MR. Thrombophilia: genetic polymorphisms and their association with retinal vascular occlusive disease. Br J Ophthalmol 2001; 85: 883-6.

16. Hansen L, Kristensen HL, Bek T, Ingerslev J. Markers of thrombophilia in retinal vein thrombosis. Acta Ophthalmol Scand 2000; 78: 523-8.

17. Greven CM, Weaber RG, Owen J. Protein S deficiency and bilateral branch retinal artery occlusion. Ophthalmology 1991; 98: 33-4.

18. Greiner K, Hafner G, Dick B, Peetz D, Prellwitz W. Retinal vascular occlusion and deficiencies in the protein C pathway. Am J Ophthalmol 1999; 128: 69-74.

19. Pereira J, Quiroga T, Goycolea M, Hidalgo P, Kaltwasser G, Mezzano D. Resistencia a la proteína $\mathrm{C}$ activada: estudio de laboratorio y prevalencia del defecto en la población chilena. Rev Méd Chile 1996; 124: 663-8.

20. Larsson J, Himarp A, Olafsdottir E, Bauer B. Activated protein $C$ resistance and anticoagulant proteins in young adults with central retinal vein occlusion. Acta Ophthalmol Scand 1999; 77: 6347.

21. Johnson MT, EL-Defrawy S, Hodge WG, Leonard BC, Kertes PJ, Taylor S et al. Prevalence of factor $\mathrm{V}$ Leiden and activated protein $\mathrm{C}$ resistance in central retinal vein occlusion. Retina 2001; 21: 161-6.

22. Ciardelia AP, Yanuzzi LA, Freund KB, Dimichele D, Nejat M, De Rosa J. Factor V Leiden, activated protein $\mathrm{C}$ resistance and retinal vein occlusion. Retina 1998; 18: 308-15.

23. Incorvaia $C$, Lamberti $G$, Parmeggiani F, Ferraresi P, Calzolari E, Bernardi F et al. Idiophatic central retinal vein occlusion in a thrombophilic patient with the heterozygous $20210 \mathrm{G} / \mathrm{A}$ prothrombin genotype. Am J Ophthalmol 1999; 128: 247-8.

24. Boyd S, Owens D, Gin T, Bunce K, Sherafat H, PerRY D ET al. Plasma homocysteine, methylene tetrahydrofolate reductase C677T and factor II G20210A polymorphisms, factor VIII, and VWF in central retinal vein occlusion. Br J Ophthalmol 2001; 85: 1313-5.

25. Glueck CH, Bell H, Vadiamani L, Gupta A, Fontaine R, Wang P. Heritable thrombophilia and hypofibrinolysis: possible causes of retinal vein occlusion. Arch Ophthalmol 1999; 117: 43-9.

26. Lip P, BLANn AD, Jones AF, Lip G. Abnormalities in haemorheological factors and lipoprotein (a) in retinal vascular occlusion: implications for increased vascular risk. Eye 1998; 12: 245-51.

27. Van Tieburg NH, Rosendal FR, Bertina RM. Thrombin activable fibrinolysis inhibitor and the risk for deep vein trombosis. Blood 200?; 95: 2855-9.

28. Pianka P, Almong Y, Man O, Goldstein M, Seal B, LoEWENSTIEN A. Hyperhomocysteinemia in patients with nonarteritic anterior ischemic optic neuropathy, central retinal artery occlusion, and central retinal vein occlusion. Ophthalmology 2000; 107: 1588-92.

29. Cahill M, Karabatzki M, Meleady R, Refsum H, UELAND P. Raised plasma homocysteine as a risk factor for retinal vascular occlusive disease. $\mathrm{Br} \mathrm{J}$ Ophthalmol 2000; 84: 154-7.

30. Jacques PF, Bostom AG, Wiшums RR, Euison RC, EсKFELDT JH. Relation between folate status, a common mutation in methylenetetrahydrofolate reductase, and plasma homocysteine concentrations. Circulation 1996; 93: 7-9.

31. Wenzier EM, Rademakers A, Boers G, Cruysberg J, WEBERS CA. Hyperhomocysteinemia in retinal artery and retinal vein occlusion. Am J Ophthalmol 1993; 115: 162-7.

32. Juhan-Vague I, Alessi MC, Mavri A, Morange PE. Plasminogen activator inhibitor-1, inflammation, obesity, insuline resistance and vascular risk. J Thromb Haemost 2003; 1: 1575-9.

33. Mils JD, GRANT PT. Insuline resistance, haemostatic factors and cardiovascular risk. Br J Diabetes Vasc Dis 2002; 2: 19-26.

34. Fong AC, Schatz H, Mc Donald HR, Burton TC, MABERLEY AL, JofFe L. Central retinal vein occlusion in young adults (papillophlebitis). Retina 1992; 12: 3-11. 
35. VINE AK, SAMAma MM. Screening for resistance to activated protein $\mathrm{C}$ and the mutant gene for factor V: Q506 in patients with central retinal vein occlusion. Am J Ophthalmol 1997; 124: 673-6.

36. Gottleb JL, BLCe JP, Mesticheш B. Activated protein $\mathrm{C}$ resistance, factor $\mathrm{V}$ leiden, and central retinal vein occlusion in young adults. Arch Ophthalmol 1998; 116: 577-9.

37. Bandello F, D’Angelo S, Parlavecchia M, Tavola A, Delia Valle P, Brancato R et al. Hypercoagulabili- ty and high lipoprotein (a) levels in patients with central retinal vein occlusion. Thromb Haemost 1994; 72: 39-43.

38. MANUCCI PM. The meassurement of multifactorial thrombophilia. Thromb Haemost 2002; 88: 1-2.

Agradecimientos:

División Diagnóstica Laboratorio Abbott. 